\title{
A stability result for girth-regular graphs with even girth
}

\author{
György Kiss*
}

\author{
$\check{S}_{\text {Stefko Miklavič }}^{\dagger}$
}

September 10, 2020

\author{
Tamás Szőnyi $\ddagger$
}

\begin{abstract}
Let $\Gamma$ denote a finite, connected, simple graph. For an edge $e$ of $\Gamma$ let $n(e)$ denote the number of girth-cycles containing $e$. For a vertex $v$ of $\Gamma$ let $\left\{e_{1}, e_{2}, \ldots, e_{k}\right\}$ be the set of edges incident to $v$ orderd such that $n\left(e_{1}\right) \leq n\left(e_{2}\right) \leq \cdots \leq n\left(e_{k}\right)$. Then $\left(n\left(e_{1}\right), n\left(e_{2}\right), \ldots, n\left(e_{k}\right)\right)$ is called the signature of $v$. The graph $\Gamma$ is said to be girth-regular, if all of its vertices have the same signature.

Let $\Gamma$ be a girth-regular graph with girth $g=2 d$ and signature $\left(a_{1}, a_{2}, \ldots, a_{k}\right)$. It is known that in this case we have $a_{k} \leq(k-1)^{d}$. In this paper we show that if $a_{k}=(k-1)^{d}-\epsilon$ for some non-negative integer $\epsilon<k-1$, then $\epsilon=0$.
\end{abstract}

\section{Introduction}

Extremal graph theory is among the early and fast-growing branches of graph theory. It was highly influenced by Erdős and Turán [18]. In a typical question of this area we fix a graph parameter (for example, in case of Turán type problems, the number of vertices), assume some property of the (simple) graphs considered (in the example, that it does not contain subgraphs $H_{1}, H_{2}, \ldots$ ) and ask for the extremal values of another graph parameter (in the example the number of edges). Our results belong to this branch of graph theory. Without going into detail about such problems in general, we just refer to a book by Bollobás [3] and a recent survey paper by Füredi and Simonovits [7]. The problems motivating our results are about cages (and the degree/diameter problem), see the dynamic surveys [5] and [16]. In this case the fixed parameter is the valency (degree)

*This research was supported in part by the Hungarian National Research, Development and Innovation Office OTKA grant no. SNN 132625, by the HAS Slovenian-Hungarian bilateral research project "Graph Colourings and Finite Geometries" (NKM-95/2019000206), and by the Slovenian Research Agency (research project J1-9110).

${ }^{\dagger}$ This research was supported in part by the Slovenian Research Agency (research program P1-0285 and research projects N1-0032, N1-0038, J1-5433, and J1-6720).

$\ddagger$ This research was supported in part by the Hungarian National Research, Development and Innovation Office OTKA grant no. K 124950, by the HAS Slovenian-Hungarian bilateral research project "Graph Colourings and Finite Geometries" (NKM-95/2019000206), and by the Slovenian Research Agency (research project J1-9110). 
of a vertex, so only regular graphs are considered, the graph property is that the girth (length of the smallest cycle) is at least $g$, and the parameter we are interested in is the minimum number of vertices. This problem was probably posed first by Kárteszi [10], with some additional property (namely, Hamiltonicity). The case of even girth is also related to finite geometries.

Recently a new type of regularity was defined and investigated by Jajcay, Kiss and Miklavič [9]. It is about the number of cycles of length $g$ (the girth) containing a given edge. A weakening of edge-girth regularity was introduced by Potočnik and Vidali [17]. Assuming some weaker regularity condition (see below in more detail) they proved an upper bound on the number of cycles of length $g$ containing an edge. We extend their results in the spirit of stability theorems. When we have an upper bound on a graph parameter, it is natural to ask what happens if it is somewhat smaller than the upper bound. In many cases there is a gap, meaning that if the parameter is smaller than the upper bound, then it is actually considerably smaller. We prove such a result. In case of a general stability result, we can go even further and prove that whenever the value of the parameter is close to the upper bound then the graph can be obtained from the extremal one by "small local modifications", that is by adding/deleting a small number of edges/vertices, see [18].

\section{Definitions and basic properties}

In this section we collect basic notation and terminology. We also give some simple, important properties of girth-regular graphs.

Let $\Gamma$ denote a finite, connected, simple graph with vertex set $V=V(\Gamma)$ and edge set $E=E(\Gamma)$. Let $d$ denote the minimal path-length distance function of $\Gamma$ and let $D=\max \{d(v, w) \mid v, w \in V\}$ denote the diameter of $\Gamma$. For $v \in V$ and an integer $i$ we let $\Gamma_{i}(v)=\{w \in V \mid d(v, w)=i\}$. We abbreviate $\Gamma(v)=\Gamma_{1}(v)$ and observe that $\Gamma_{i}(v)=\emptyset$ whenever $i<0$ or $i>D$. For an edge $u v$ of $\Gamma$, let $D_{j}^{i}(u, v)=\Gamma_{i}(u) \cap \Gamma_{j}(v)$.

We say that $\Gamma$ is regular with valency $k(k \in \mathbb{Z})$, whenever $|\Gamma(v)|=k$ for every $v \in V$. The girth $g$ of $\Gamma$ is the length of a shortest cycle in $\Gamma$. If $C$ is a cycle of $\Gamma$ of girth length $g$, then we refer to $C$ as a girth-cycle of $\Gamma$. The following definition was introduced in [17].

Definition 2.1. Let $\Gamma$ be a k-regular graph. For an edge e of $\Gamma$ let $n(e)$ denote the number of girth-cycles containing e. For a vertex $v$ of $\Gamma$ let $\left\{e_{1}, e_{2}, \ldots, e_{k}\right\}$ be the set of edges incident to $v$ orderd such that $n\left(e_{1}\right) \leq n\left(e_{2}\right) \leq \cdots \leq n\left(e_{k}\right)$. Then $\left(n\left(e_{1}\right), n\left(e_{2}\right), \ldots, n\left(e_{k}\right)\right)$ is called the signature of $v$. The graph $G$ is said to be girth-regular, if all of its vertices have the same signature.

As mentioned in the introduction, girth regularity generalizes edge-girth regularity introduced in [9]. For an edge-girth regular graph $a_{1}=a_{2}=\ldots=a_{k}$. The following central question about girth-regular graphs was proposed in [17]:

Question 2.2. Given integers $k$ and $g$, for which $k$-tuples $\sigma=\left(a_{1}, a_{2}, \ldots, a_{k}\right) \in \mathbb{Z}^{k}$ does a girth-regular graph of girth $g$ and signature $\sigma$ exist? 
Although the above question seems to be very difficult in its full generality, an upper bound on the entries $a_{i}$ of the signature in terms of the valence $k$ and the girth $g$ was given in [17, Theorem 1.2]:

Proposition 2.3. Let $\Gamma$ be a girth-regular graph with girth $g$ and signature $\left(a_{1}, a_{2}, \ldots, a_{k}\right)$. Then $a_{k} \leq(k-1)^{d}$, where $d=\lfloor g / 2\rfloor$.

In this paper we push the above result a bit further in the case when $g$ is even. Namely, we study girth-regular graphs with girth $g=2 d$ and signature $\left(a_{1}, a_{2}, \ldots, a_{k}\right)$, where $a_{k} \leq(k-1)^{d}-\epsilon$ for some small values of $\epsilon$. The following theorem is the main result of this paper.

Theorem 2.4. Let $\Gamma$ be a girth-regular graph with girth $g=2 d$ and signature $\left(a_{1}, a_{2}, \ldots, a_{k}\right)$, such that $a_{k}=(k-1)^{d}-\epsilon$ for some non-negative integer $\epsilon<k-1$. Then $\epsilon=0$ and $\Gamma$ is the incidence graph of a finite generalized $d$-gon of order $(k-1, k-1)$. (Hence $d \in\{2,3,4,6,8\}$.)

The following straightforward observation will be used through the rest of the paper frequently without explicitly referring to it (see also [17, Subsection 2.2]).

Proposition 2.5. Let $\Gamma$ be a graph with girth $2 d, d \geq 2$. Let uv be an edge of $\Gamma$ and let $D_{j}^{i}=D_{j}^{i}(u, v)$. Then the following hold.

(i) If $x, y$ are vertices of $\Gamma$ with $d(x, y) \leq d-1$, then there is a unique path of length $d(x, y)$ between $x$ and $y$.

(ii) $D_{i}^{i}=\emptyset$ for $1 \leq i \leq d-1$.

(iii) For $1 \leq i \leq d-1$ and for $z \in D_{i+1}^{i}$ (resp. $z \in D_{i}^{i+1}$ ), we have that $\left|\Gamma(z) \cap D_{i}^{i-1}\right|=1$ (resp. $\left.\left|\Gamma(z) \cap D_{i-1}^{i}\right|=1\right)$.

(iv) If $\Gamma$ is regular with valency $k$, then for $0 \leq i \leq d-2$ and for $z \in D_{i+1}^{i}$ (resp. $\left.z \in D_{i}^{i+1}\right)$, we have that $\left|\Gamma(z) \cap D_{i+2}^{i+1}\right|=k-1$ (resp. $\left.\left|\Gamma(z) \cap D_{i+1}^{i+2}\right|=k-1\right)$.

(v) If $\Gamma$ is regular with valency $k$, then for $0 \leq i \leq d-1$ we have that

$$
\left|D_{i+1}^{i}\right|=\left|D_{i}^{i+1}\right|=(k-1)^{i} .
$$

(vi) If $\Gamma$ is regular with valency $k$ and $n(u v)=(k-1)^{d}-\epsilon$, then there are exactly $(k-1)^{d}-\epsilon$ edges between $D_{d}^{d-1}$ and $D_{d-1}^{d}$.

Keeping in mind Proposition 2.5, the proof of the following lemma is straightforward.

Lemma 2.6. Let $\Gamma$ be a k-regular graph with girth $g=2 d$, and let $u_{1} u_{2}$ and $v_{1} v_{2}$ be two edges of $\Gamma$. Without loss of generality we may assume that $d\left(u_{1}, v_{1}\right)=\min \left\{d\left(u_{i}, v_{j}\right): 1 \leq\right.$ $i, j \leq 2\}$. Suppose that there are $0 \leq \alpha_{u}$ forbidden edges through $u_{2}, \alpha_{v}$ forbidden edges through $v_{2}$, and that $\alpha_{u} \leq \alpha_{v}$. Let $m=d\left(u_{1}, v_{1}\right)+1$, and let $c$ denote the maximum 


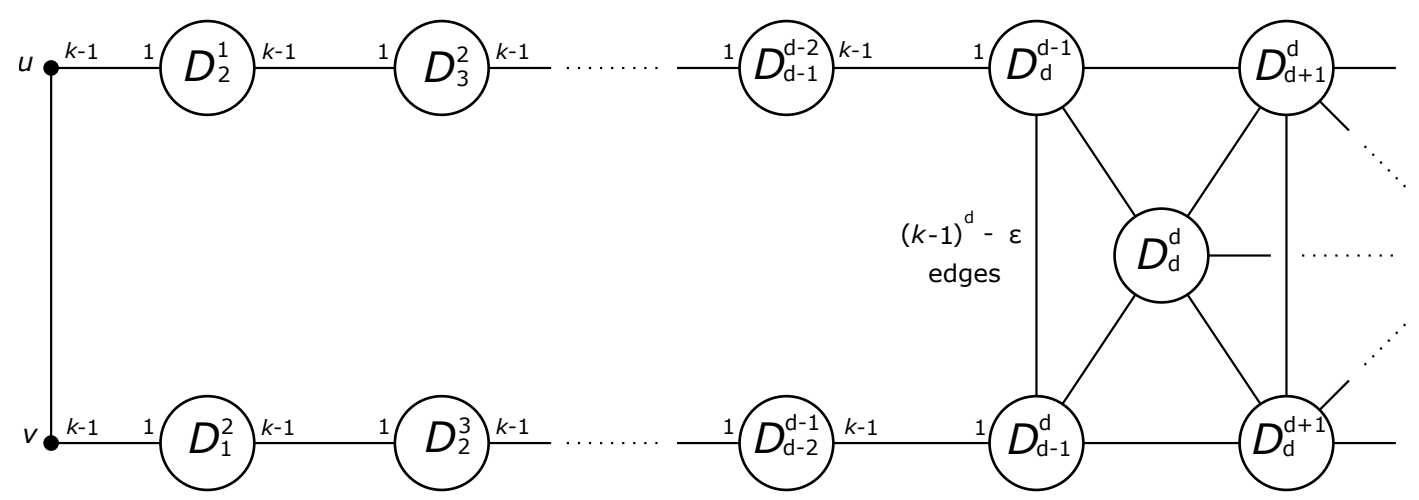

Figure 1: A regular graph with valency $k$ and girth $2 d$, where $n(u v)=(k-1)^{d}-\epsilon$. The numbers near the bubble representing the set $D_{j}^{i}$ represent the number of neighbours, that each vertex of $D_{j}^{i}$ has in the neighbouring bubble.

number of girth-cycles containing both $u_{1} u_{2}$ and $v_{1} v_{2}$, but not containing any forbidden edge. Then

$$
c \leq \begin{cases}\left(k-1-\alpha_{u}\right)\left(k-1-\alpha_{v}\right)(k-1)^{d-m-2}, & \text { if } m \leq d-2, \\ k-1-\alpha_{v}, & \text { if } m=d-1, \\ 1, & \text { if } m=d, \\ 0, & \text { if } m \geq d+1 .\end{cases}
$$

Observe that cycles are the only girth-regular graphs for $k=2$ and their signature is $(1,1)$, so in the rest of this paper we assume $k \geq 3$ and we will use the following notation.

Notation 2.7. Let $\Gamma$ be a girth-regular graph with valency $k \geq 3$, girth $g=2 d$ and signature $\left(a_{1}, a_{2}, \ldots, a_{k}\right)$. Suppose that $a_{k}=(k-1)^{d}-\epsilon$ for some $\epsilon<k-1$. Let uv be an edge with $n(u v)=a_{k}$, and let $D_{j}^{i}=D_{j}^{i}(u, v)$. Note that $D_{i}^{i}=\emptyset$ for $1 \leq i \leq d-1$ and that there are no edges between $D_{i}^{i-1}$ and $D_{i-1}^{i}$ for $1 \leq i \leq d-1$.

If $\epsilon \geq 1$ then we let $\mathcal{E}$ denote the $2 \epsilon$ edges of $\Gamma$, that have one endpoint in $D_{d}^{d-1} \cup D_{d-1}^{d}$, and the other endpoint in $D_{d+1}^{d} \cup D_{d}^{d+1} \cup D_{d}^{d}$. Note that $\epsilon$ of these edges have one endpoint in $D_{d}^{d-1}$, while the other $\epsilon$ of these edges have endpoint in $D_{d-1}^{d}$. For every $r \in D_{d-1}^{d}$ $\left(b \in D_{d}^{d-1}\right.$, respectively) we let $h(r)=k-1-\left|\Gamma(r) \cap D_{d}^{d-1}\right|\left(h(b)=k-1-\left|\Gamma(b) \cap D_{d-1}^{d}\right|\right.$, respectively). Let $\left\{r_{1}, r_{2}, \ldots, r_{m}\right\} \subseteq D_{d-1}^{d}$ be the set of vertices of $D_{d-1}^{d}$, for which the value of the function $h$ is positive. Choose the indices in such a way that $h\left(r_{i}\right) \leq h\left(r_{j}\right)$ for $i<j$. Similarly, let $\left\{b_{1}, b_{2}, \ldots, b_{n}\right\} \subseteq D_{d}^{d-1}$ be the set of vertices of $D_{d}^{d-1}$, for which the value of the function $h$ is positive. Again, choose the indices in such a way that $h\left(b_{i}\right) \leq h\left(b_{j}\right)$ for $i<j$. We may assume without loss of generality that $\gamma=h\left(r_{m}\right) \leq h\left(b_{n}\right)=\beta$. Let $\mu=h\left(r_{1}\right)$ and $\nu=h\left(b_{1}\right)$.

With reference to Notation 2.7, observe that by definition we have

$$
\sum_{r \in D_{d-1}^{d}} h(r)=\sum_{i=1}^{m} h\left(r_{i}\right)=\sum_{b \in D_{d}^{d-1}} h(b)=\sum_{i=1}^{n} h\left(b_{i}\right)=\epsilon .
$$


There is at most one missing edge between any pair of vertices $r \in D_{d-1}^{d}$ and $b \in D_{d}^{d-1}$, hence $m \geq \beta$ and $n \geq \gamma$. These observations obviously imply the following inequalities:

$$
\mu \beta \leq \mu m \leq \epsilon, \quad \nu \gamma \leq \nu n \leq \epsilon .
$$

Observe also that it follows from the above comments that

$$
\mu^{2} \leq \mu \gamma \leq \mu \beta \leq \mu m \leq \epsilon
$$

implying that $\mu \leq \sqrt{\epsilon}$.

First, we give a lower bound on $a_{1}$ using the vertex $u$.

Lemma 2.8. With reference to Notation 2.7 we have that

$$
a_{1} \geq(k-1)^{d-2} \max \{(k-1-\beta)(k-1)-\epsilon,(k-1-\gamma)(k-1)-\epsilon\} .
$$

Proof. We prove that $a_{1} \geq(k-1)^{d-2}((k-1-\beta)(k-1)-\epsilon)$. The proof of $a_{1} \geq$ $(k-1)^{d-2}((k-1-\gamma)(k-1)-\epsilon)$ is similar.

Recall that $n(u v)=a_{k}$ and that $D_{j}^{i}=D_{j}^{i}(u, v)$. Let $b \neq v$ be a neighbour of $u$ such that $n(u b)=a_{1}$. Pick $b^{\prime} \in D_{d}^{d-1} \cap \Gamma_{d-2}(b)$ and $r^{\prime} \in D_{d-1}^{d} \cap \Gamma\left(b^{\prime}\right)$ and observe that for each $\tilde{r} \in\left(\Gamma\left(r^{\prime}\right) \cap\left(D_{d}^{d-1} \cup D_{d-2}^{d-1}\right) \backslash\left\{b^{\prime}\right\}\right.$, there is a unique girth-cycle containing the arc $u b$ and the 2-arc $b^{\prime} r^{\prime} \tilde{r}$. Abbreviate $A=D_{d}^{d-1} \cap \Gamma_{d-2}(b)$ and $B\left(b^{\prime}\right)=D_{d-1}^{d} \cap \Gamma\left(b^{\prime}\right)$, where $b^{\prime} \in A$. Note that $|A|=(k-1)^{d-2}$, and so, by (1), we have

$$
\begin{aligned}
a_{1} & =n(u b) \geq \sum_{b^{\prime} \in A} \sum_{r^{\prime} \in B\left(b^{\prime}\right)}\left(k-1-h\left(r^{\prime}\right)\right) \\
& =\sum_{b^{\prime} \in A} \sum_{r^{\prime} \in B\left(b^{\prime}\right)}(k-1)-\sum_{b^{\prime} \in A} \sum_{r^{\prime} \in B\left(b^{\prime}\right)} h\left(r^{\prime}\right) \\
& \geq(k-1) \sum_{b^{\prime} \in A}\left(k-1-h\left(b^{\prime}\right)\right)-\sum_{b^{\prime} \in A} \epsilon \\
& \geq(k-1) \sum_{b^{\prime} \in A}(k-1-\beta)-(k-1)^{d-2} \epsilon \\
& =(k-1)(k-1)^{d-2}(k-1-\beta)-(k-1)^{d-2} \epsilon \\
& =(k-1)^{d-2}((k-1)(k-1-\beta)-\epsilon) .
\end{aligned}
$$

\section{The case $g=4$}

In this section we consider the case $g=4$.

Lemma 3.1. With reference to Notation 2.7, assume that $g=4$ and $\epsilon \geq 1$. Then $n \geq 2$ and $m \geq 2$. 
Proof. We prove that $n \geq 2$. The proof that $m \geq 2$ is similar. Suppose on the contrary that $n=1$. Note that in this case $\beta=\epsilon, \gamma=1, m=\epsilon$ and $h\left(r_{i}\right)=1$ for $1 \leq i \leq m$. Let $w$ be a unique vertex, such that $r_{1} w \in \mathcal{E}$. Let $t=\left|\Gamma(w) \cap D_{1}^{2}\right|$ and note that $t \leq \epsilon$. Note that girth-cycles containing edge $r_{1} w$ are exactly cycles of the form $\left(w, r_{1}, x, y, w\right)$, where $x \in\{v\} \cup\left(D_{2}^{1} \backslash\left\{b_{1}\right\}\right)$ and $y \in\left(\Gamma(w) \cap D_{1}^{2}\right) \backslash\left\{r_{1}\right\}$. Therefore, $n\left(r_{1} w\right)=(k-1)(t-1) \leq$ $(k-1)(\epsilon-1)$. Therefore, since $\gamma=1$, we have by Lemma 2.8 that

$$
(k-2)(k-1)-\epsilon \leq a_{1} \leq n\left(r_{1} w\right) \leq(k-1)(\epsilon-1) .
$$

It follows that $(k-1)^{2} \leq k \epsilon$, and so

$$
k-2+\frac{1}{k} \leq \epsilon<k-1
$$

contradicting the fact that $\epsilon$ is an integer.

We now give an upper bound for $a_{1}$.

Lemma 3.2. With reference to Notation 2.7, assume that $g=4$ and $\epsilon \geq 1$. Let $\alpha=$ $h\left(b_{n-1}\right)$. Then

$$
a_{1} \leq \epsilon+(\epsilon-\beta)(k-1)-(\alpha-1)(\epsilon-\alpha-\beta+1)
$$

Proof. Let $\left\{w_{1}, \ldots, w_{\alpha}\right\}=\Gamma\left(b_{n-1}\right) \backslash\left(\{u\} \cup D_{1}^{2}\right)$. We estimate $n\left(b_{n-1} w_{1}\right)$. To do this we split the girth-cycles $\left(w_{1}, b_{n-1}, x, y, w_{1}\right)$ into two types, depending on vertex $x$. We say that the girth-cycle is of type 1 if $x \in\left\{w_{2}, \ldots, w_{\alpha}\right\}$, and of type 2 if $x \in\{u\} \cup D_{1}^{2}$. By Lemma 2.6 there are at most $(\alpha-1)(k-1)$ girth-cycles of type 1 . To estimate the number of girth-cycles of type 2 , we further split these girth-cycles into two subfamilies, depending on vertex $y$. Let us say that the girth-cycle $\left(w_{1}, b_{n-1}, x, y, w_{1}\right)$ with $x \in\{u\} \cup D_{1}^{2}$ is of type $2 \mathrm{a}$ if $y \in D_{2}^{1}$, and of type $2 \mathrm{~b}$ if $y \notin D_{2}^{1}$. If the girth-cycle is of type $2 \mathrm{~b}$, then $x \in D_{1}^{2}$ and $x y$ is one of the edges from $\mathcal{E}$. There are $\epsilon$ of such edges, and for each of these edges there is, by Lemma 2.6, at most one girth-cycle, containing also the edge $w_{1} b_{n-1}$. Therefore, there are at most $\epsilon$ girth-cycles of type $2 \mathrm{~b}$. To estimate the number of girth-cycles of type $2 \mathrm{a}$, observe that $b_{n-1}$ has $k-\alpha$ neighbours in $\{u\} \cup D_{1}^{2}$, and that $w_{1}$ has at most $\epsilon-\alpha-\beta+1$ neighbours in $D_{2}^{1} \backslash\left\{b_{n-1}\right\}$. This shows that the number of girth-cycles of type $2 \mathrm{a}$ is at most $(k-\alpha)(\epsilon-\alpha-\beta+1)$. Therefore,

$$
\begin{aligned}
a_{1} \leq n\left(b_{n-1} w_{1}\right) & \leq(\alpha-1)(k-1)+\epsilon+(k-\alpha)(\epsilon-\alpha-\beta+1) \\
& =\epsilon+(\epsilon-\beta)(k-1)-(\alpha-1)(\epsilon-\alpha-\beta+1) .
\end{aligned}
$$

Lemma 3.3. With reference to Notation 2.7, assume that $g=4$ and $\epsilon \geq 1$. Then $\epsilon=k-2$ and $\beta=\gamma$.

Proof. By Lemmas 2.8 and 3.2 we get that

$$
((k-1)-\gamma)(k-1)-\epsilon \leq \epsilon+(\epsilon-\beta)(k-1)-(\alpha-1)(\epsilon-\alpha-\beta+1),
$$

that is,

$$
(k-1)^{2}+(\beta-\gamma)(k-1) \leq(k+1) \epsilon-(\alpha-1)(\epsilon-\alpha-\beta+1) .
$$


Taking into account that $\gamma \leq \beta$, that $\alpha \geq 1$ and that $\alpha+\beta \leq \epsilon$, inequality (6) implies that

$$
(k-1)^{2} \leq(k+1) \epsilon-\alpha+1 \leq(k+1) \epsilon,
$$

and so $\epsilon>k-3$. As $\epsilon<k-1$, we have that $\epsilon=k-2$ as claimed. Now if $\beta-\gamma \geq 1$, then inequality (6) (together with $\epsilon=k-2$ ) implies that

$$
(\alpha-1)(k-\alpha-\beta-1) \leq-2<0,
$$

forcing that $k-\alpha-\beta-1 \leq-1$. But this implies $k \leq \alpha+\beta \leq \epsilon=k-2$, a contradiction. Therefore, $\beta=\gamma$ as claimed.

Lemma 3.4. With reference to Notation 2.7, assume that $g=4$ and $\epsilon \geq 1$. Then the following hold.

(i) $\nu=\mu=1$;

(ii) $h\left(b_{i}\right)=h\left(r_{j}\right)=1$ for $1 \leq i \leq n-1,1 \leq j \leq m-1$;

(iii) $n=m$.

Proof. (i) We prove that $\nu=1$. The proof that $\mu=1$ is similar. Suppose to the contrary that $\nu \geq 2$ and consider vertex $b_{1}$. Let $\left\{w_{1}, \ldots, w_{\nu}\right\}=\Gamma\left(b_{1}\right) \backslash\left(\{u\} \cup D_{1}^{2}\right)$. Without loss of generality we could assume that $\left|\Gamma\left(w_{1}\right) \cap\left\{b_{2}, \ldots, b_{n-1}\right\}\right|$ is minimal among $w_{1}, w_{2}, \ldots, w_{\nu}$. As in the proof of Lemma 3.2 split the girth-cycles containing the edge $b_{1} w_{1}$ into three families: girth-cycles of type 1, of type $2 \mathrm{a}$ and of type $2 \mathrm{~b}$. A similar argument as in the proof of Lemma 3.2 shows that there are at most $(\nu-1)(k-1)$ girth-cycles of type 1 , and at most $\epsilon=k-2$ girth-cycles of type $2 \mathrm{~b}$.

To estimate the number of girth-cycles of type $2 \mathrm{a}$, observe first that $b_{1}$ has $k-\nu$ neighbours in $\{u\} \cup D_{1}^{2}$. Let us now estimate the number of neigbours of $w_{1}$ in $D_{2}^{1} \backslash\left\{b_{1}\right\}$. Observe that there are at most $\epsilon-\nu-\beta$ edges between the sets $\left\{b_{2}, \ldots, b_{n-1}\right\}$ and $\left\{w_{1}, \ldots, w_{\nu}\right\}$. As $\nu \geq 2$, vertex $w_{1}$ is not an endpoint of at least half of these edges. This shows that $w_{1}$ has at most $(\epsilon-\nu-\beta) / 2+1$ neighbours in $D_{2}^{1} \backslash\left\{b_{1}\right\}$ (recall that $w_{1}$ could be adjacent also with $b_{n}$ ), and so the number of girth-cycles of type $2 \mathrm{a}$ is at most

$$
(k-\nu)\left(\frac{\epsilon-\nu-\beta}{2}+1\right)=(k-\nu)\left(\frac{k-2-\nu-\beta}{2}+1\right) \text {. }
$$

Therefore, as $\nu+\beta \leq \epsilon=k-2$ and $\nu \geq 2$, we have

$$
\begin{aligned}
a_{1} \leq n\left(b_{1} w_{1}\right) & \leq(\nu-1)(k-1)+(k-\nu)\left(\frac{k-2-\nu-\beta}{2}+1\right)+k-2 \\
& \leq \nu(k-1)-\frac{\nu+\beta}{2}(k-2)+\frac{k^{2}-2 k-2}{2} .
\end{aligned}
$$

Combining inequalities (3) and (7) we obtain

$$
(k-1)^{2}-\beta(k-1)-(k-2) \leq \nu(k-1)-\frac{\nu+\beta}{2}(k-2)+\frac{k^{2}-2 k-2}{2},
$$


which is equivalent to $k^{2}-4 k+8 \leq(\mu+\beta) k$. By (2), we have that $2 \beta \leq \nu \beta \leq k-2$, and so $\nu+\beta \leq(k-2) / \beta+\beta \leq 2+(k-2) / 2$. Combining this with the above inequality we get that

$$
k^{2}-4 k+8 \leq\left(2+\frac{k-2}{2}\right) k,
$$

and so $k \leq 8$. On the other hand, by (2), we have that $4 \leq \nu \beta \leq \epsilon=k-2$, and so $k \geq 6$. Furthermore, observe that $\nu \beta \leq k-2$ together with (1) nad Lemma 3.3 imply that $\nu=\beta=\gamma=2$ and $k \in\{6,8\}$. We now analyze these cases separately.

Case 1: $k=8$. Lemma 2.8 gives us $a_{1} \geq 29$, while (7) gives us $a_{1} \leq 25$, a contradiction. Case 2: $k=6$. Let $D_{2}^{1}=\left\{b_{1}, b_{2}, b_{3}, b_{4}, b_{5}\right\}$, such that $h\left(b_{1}\right)=h\left(b_{2}\right)=2$ and $h\left(b_{3}\right)=$ $h\left(b_{4}\right)=h\left(b_{5}\right)=0$. Note that, by Lemma 3.2, we have that $a_{1} \leq 13$. Observe that $n(u v)=n\left(u b_{3}\right)=n\left(u b_{4}\right)=n\left(u b_{5}\right)=21=a_{6}$, and so every vertex $x$ of $\Gamma$ has at least four neighbours $y$ with $n(x y)=21$. Furthermore, we could assume without loss of generality that $a_{1}=n\left(u b_{1}\right)$.

We will now show that $\mu=2$. Recall first that $\gamma=2$ and so $h(r) \leq 2$ for every $r \in D_{1}^{2}$. Suppose to the contrary that $\mu=1$, which implies that there are vertices $r_{1}, r_{2}, r_{3} \in D_{1}^{2}$ with $h\left(r_{1}\right)=h\left(r_{2}\right)=1$ and $h\left(r_{3}\right)=2$, while for any other vertex $r \in D_{1}^{2}$ we have $h(r)=0$.

Observe that $n\left(v r_{3}\right) \leq 17<21$. As $v$ has at least four neighbours $y$ with $n(v y)=21$, we have $n(v r)=21$ for at least one $r \in\left\{r_{1}, r_{2}\right\}$. Pick such $r$ and let $w$ be the unique neighbour of $r$, which is not contained in $\{v\} \cup D_{2}^{1}$. Observe that $r$ is adjacent with $b_{3}, b_{4}, b_{5}$ and exactly one of $b_{1}, b_{2}$, say with $b_{1}$. Let us now count girth-cycles $(v, r, x, y, v)$. If $x \in\left\{b_{3}, b_{4}, b_{5}\right\}$, then there are 5 such cycles, while if $x=b_{1}$, there are 3 such cycles. In addition, there are at most 2 such cycles in case $x=w$ : possibly one with $y=r_{3}$ and one with $y=r^{\prime}$, where $\left\{r^{\prime}\right\}=\left\{r_{1}, r_{2}\right\} \backslash\{r\}$. This gives us at most 20 girth-cycles containing $v r$, contradicting $n(v r)=21$. This shows that $\mu=2$.

It follows that there are three vertices $r, r^{\prime}, r^{\prime \prime} \in D_{1}^{2}$ with $h(r)=h\left(r^{\prime}\right)=h\left(r^{\prime \prime}\right)=0$, and so $b_{1}$ is adjacent with each of these three vertices. But this implies that there are at least $5+5+5$ girth-cycles containing edge $u b_{1}$, contradicting $n\left(u b_{1}\right)=a_{1} \leq 13$.

(ii) We prove that $h\left(b_{i}\right)=1$ for $1 \leq i \leq n-1$. The proof that $h\left(r_{j}\right)=1$ for $1 \leq j \leq m-1$ is similar. Note that to prove that $h\left(b_{i}\right)=1$ for $1 \leq i \leq n-1$ it is enough to prove that $h\left(b_{n-1}\right)=1$. Suppose on the contrary that $h\left(b_{n-1}\right) \geq 2$. Recall that $\nu=h\left(b_{1}\right)=1$ by (i) above. Let $w_{1}$ be the only neighbour of $b_{1}$, which is not contained in $\{u\} \cup D_{1}^{2}$. Consider the girth-cycles through $b_{1} w_{1}$. As in (i) above we split these cycles into three families: cycles of type 1 , cycles of type $2 \mathrm{a}$ and cycles of type $2 \mathrm{~b}$. There are at most $\epsilon=k-2$ girth-cycles of type $2 \mathrm{~b}$, and as $\nu=1$, there are no girth-cycles of type 1 .

To estimate the number of girth-cycles of type $2 \mathrm{a}$, observe first that $b_{1}$ has $k-1$ neighbours in $\{u\} \cup D_{1}^{2}$, while $w_{1}$ has at most

$$
\epsilon-1-\left(h\left(b_{n-1}\right)-1\right)-(\beta-1)=k-1-\beta-h\left(b_{n-1}\right) \leq k-3-\beta
$$

neighbours in $D_{2}^{1} \backslash\left\{b_{1}\right\}$. It follows that there are at most

$$
k-2+(k-1)(k-3-\beta)
$$

girth-cycles containing $b_{1} w_{1}$. Therefore $a_{1} \leq n\left(b_{1} w_{1}\right) \leq k-2+(k-1)(k-3-\beta)$, contradicting Lemma 2.8. 
(iii) This follows from (ii) above, Equation (1) and Lemma 3.3.

Lemma 3.5. With reference to Notation 2.7, assume that $g=4$ and $\epsilon \geq 1$. Then $\beta=1$.

Proof. Recall that $\epsilon=k-2$ and that $h\left(b_{i}\right)=h\left(r_{i}\right)=1$ for $1 \leq i \leq n-1$. It follows that $n-1+\beta=k-2$. Pick $b \in D_{2}^{1}$ with $h(b)=0$. It is easy to see that $n(u b)=$ $(k-1)^{2}-k+2=k^{2}-3 k+3$, and so for at least $k-n=\beta+1$ neighbours $b$ of $u$ we have that $n(u b)=a_{k}=k^{2}-3 k+3$. As $\Gamma$ is girth-regular, it follows that also $b_{n}$ must have at least $\beta+1$ neighbours $x$ with $n\left(b_{n} x\right)=a_{k}$. In the rest of the proof we estimate $n\left(b_{n} x\right)$ for various neighbours $x$ of $b_{n}$. We omit the details and leave them to a reader.

It is straightforward to see that for $x=u$ we have

$$
n\left(b_{n} u\right) \leq(n-1)+(k-\beta-1)(k-1)=k^{2}-k-1-\beta k,
$$

and so $n\left(b_{n} u\right)<a_{k}$ if $\beta \geq 2$. Assume now that $b_{n}$ is adjacent with $r \in D_{1}^{2}$ with $h(r)=0$. Similarly as above we find that

$$
n\left(b_{n} r\right) \leq(n-1)+(k-\beta-1)(k-1),
$$

and so $n\left(b_{n} r\right)<a_{k}$ if $\beta \geq 2$. If $b_{n}$ is adjacent with $r_{j}(1 \leq i \leq n-1)$, then

$$
n\left(b_{n} r_{j}\right) \leq \beta+(n-1)+(n-1)+(k-\beta-1)(k-2)=k^{2}-k-2+\beta-\beta k,
$$

and so again $n\left(b_{n} r_{j}\right)<a_{k}$ if $\beta \geq 2$. Assume finally that $b_{n}$ is adjacent with $r_{n}$. Then we have

$$
n\left(b_{n} r_{n}\right) \leq \beta^{2}+(n-1)+(n-1)+(k-\beta-1)(k-\beta-1)=k^{2}-3+2 \beta(\beta-k) .
$$

If $\beta \geq 2$, then $\beta(k-\beta) \geq 2(k-2)$, and so $n\left(b_{n} r_{n}\right) \leq k^{2}-3-4(k-2)<a_{k}$ as $k \geq 3$. Therefore, if $\beta \geq 2$, then $b_{n}$ has at most $\beta$ neighbours $x$ with $n\left(b_{n} x\right)=a_{k}$, contradicting the fact that $\Gamma$ is girth-regular. This shows that $\beta=1$.

Theorem 3.6. With reference to Notation 2.7, assume that $g=4$. Then $\epsilon=0$ and $\Gamma$ is the complete bipartite graph $K_{k, k}$.

Proof. Suppose on the contrary that $\epsilon \geq 1$. Recall that $\epsilon=k-2$ and $\beta=\gamma=1$ by Lemma 3.3 and Lemma 3.5. Therefore, there is exactly one vertex $r \in D_{1}^{2}$ with $h(r)=0$, and there is exactly one vertex $r_{j}(1 \leq j \leq n=k-2)$, such that $b_{1}$ is not adjacent with $r_{j}$. Without loss of generality we could assume that $j=1$. Consider now vertex $b_{1}$ and let $w_{1}$ be the unique neighbour of $b_{1}$, which is not contained in $\{u\} \cup D_{1}^{2}$. Similarly as in the proof of Lemma 3.2 we split the girth-cycles $\left(w_{1}, b_{1}, x, y, w_{1}\right)$ containing the edge $b_{1} w_{1}$ into two families. To do this first note that $x \in\{u\} \cup D_{1}^{2}$. We say that the girth-cycle $\left(w_{1}, b_{1}, x, y, w_{1}\right)$ is of type $2 \mathrm{a}$ if $y \in D_{2}^{1}$, and of type $2 \mathrm{~b}$ if then $y \notin D_{2}^{1}$. As $b_{1}$ is not adjacent with $r_{1}$, there are at most $\epsilon-1=k-3$ girth-cycles of type $2 \mathrm{~b}$. To estimate the number of girth-cycles of type $2 \mathrm{a}$, observe first that $y \in\left\{b_{2}, \ldots, b_{n}\right\}$. Let $r$ denote the unique vertex of $D_{1}^{2}$ with $h(r)=0$ and note that $u$ and $r$ are adjacent with each $b_{i}(2 \leq i \leq n)$. Let $b$ denote the unique vertex of $D_{2}^{1}$ with $h(b)=0$. Pick $r_{i}(2 \leq i \leq n)$. 
As $h\left(r_{i}\right)=1$ and $r_{i}$ is adjacent with $b$ and $b_{1}$, there exist exactly one $j(2 \leq j \leq n)$, such that $r_{i}$ and $b_{j}$ are not adjacent. The above comments shows that there are at most

$$
(k-3)+(k-3)+(k-3)(k-4)=(k-3)(k-2)
$$

girth-cycles of type 2a. This shows that

$$
a_{1} \leq n\left(b_{1} w_{1}\right) \leq k-3+(k-2)(k-3)=k^{2}-4 k+3 .
$$

On the other hand, we have $a_{1} \geq(k-2)^{2}$ by Lemma 2.8 , and so $(k-2)^{2} \leq k^{2}-4 k+3$, a contradiction. Therefore $\epsilon=0$, which implies that $\Gamma$ is a complete bipartite graph $K_{k, k}$.

\section{The case $g \geq 6$ even}

In this section we study girth-regular graphs with girth $g=2 d \geq 6$. With reference to Notation 2.7, assume that $g=2 d \geq 6$. For every $z \in D_{1}^{2}$ we define

$$
\beta(z)=\sum_{r \in D_{d-1}^{d} \cap \Gamma_{d-2}(z)} h(r) .
$$

Note that for $z \in D_{1}^{2}$ we have $\left|D_{d-1}^{d} \cap \Gamma_{d-2}(z)\right|=(k-1)^{d-2}$ and that for $z, z^{\prime} \in D_{1}^{2}\left(z \neq z^{\prime}\right)$, the sets $D_{d-1}^{d} \cap \Gamma_{d-2}(z)$ and $D_{d-1}^{d} \cap \Gamma_{d-2}\left(z^{\prime}\right)$ are disjoint as the girth of $\Gamma$ is $2 d$. Therefore,

$$
\sum_{z \in D_{1}^{2}} \beta(z)=\sum_{r \in D_{d-1}^{d}} h(r)=\epsilon .
$$

In particular, $\beta(z) \leq \epsilon$.

Lemma 4.1. With reference to Notation 2.7, assume that $g=2 d \geq 6$ and $\epsilon \geq 1$. Then

$$
a_{1} \geq(k-1)^{d}-k \epsilon .
$$

Proof. Pick $z \in D_{1}^{2}$ with $n(v z)=a_{1}$ and let $w_{1}, w_{2}, \ldots, w_{(k-1)}{ }^{d-2}$ be the vertices of $D_{d-1}^{d} \cap$ $\Gamma_{d-2}(z)$. For $1 \leq j \leq(k-1)^{d-2}$ consider the $2 d$-cycles of the form $\left(v, z, \ldots, w_{j}, b, r, r^{\prime}, \ldots\right)$ with $b \in D_{d}^{d-1}$, where $\left(v, z, \ldots, w_{j}\right)$ is the unique path from $v$ to $w_{j}$ of length $d-1$. Observe that for a fixed $w_{j}, b$ and $r$, there is only one such cycle, and that for a fixed $w_{j}, b$, we could choose $r$ in $(k-1-h(b))$ different ways. Therefore,

$$
\begin{aligned}
a_{1}=n(v z) & \geq \sum_{j=1}^{(k-1)^{d-2}} \sum_{b \in \Gamma\left(w_{j}\right) \cap D_{d}^{d-1}}(k-1-h(b)) \\
& =\sum_{j=1}^{(k-1)^{d-2}} \sum_{b \in \Gamma\left(w_{j}\right) \cap D_{d}^{d-1}}(k-1)-\sum_{j=1}^{(k-1)^{d-2}} \sum_{b \in \Gamma\left(w_{j}\right) \cap D_{d}^{d-1}} h(b) .
\end{aligned}
$$


Furthermore, observe that for a fixed $w_{j}$ we could choose $b$ in $\left(k-1-h\left(w_{j}\right)\right)$ different ways, and so

$$
\sum_{j=1}^{(k-1)^{d-2}} \sum_{b \in \Gamma\left(w_{j}\right) \cap D_{d}^{d-1}}(k-1)=(k-1) \sum_{j=1}^{(k-1)^{d-2}}\left(k-1-h\left(w_{j}\right)\right)=(k-1)^{d}-(k-1) \beta(z) .
$$

Finally, the sets $\Gamma\left(w_{j}\right) \cap D_{d}^{d-1}$ and $\Gamma\left(w_{\ell}\right) \cap D_{d}^{d-1}$ are pairwise different if $j \neq \ell$ (otherwise we would get a cycle of length $2 d-2)$, and so

$$
\sum_{j=1}^{(k-1)^{d-2}} \sum_{b \in \Gamma\left(w_{j}\right) \cap D_{d}^{d-1}} h(b) \leq \sum_{b \in D_{d}^{d-1}} h(b)=\epsilon .
$$

This, together with $\beta(z) \leq \epsilon$, shows that

$$
a_{1}=n(v z) \geq(k-1)^{d}-(k-1) \beta(z)-\epsilon \geq(k-1)^{d}-k \epsilon .
$$

Lemma 4.2. With reference to Notation 2.7, assume that $g=2 d \geq 6$ and $\epsilon \geq 1$. Then

$$
a_{1}<(k-1)^{d-2}(k \epsilon-k+2)
$$

Proof. Pick a vertex $r \in D_{d-1}^{d}$ with $h(r) \geq 1$ and abbreviate $\alpha=h(r)$. Let

$$
A=\bigcup_{i=0}^{d-1}\left(D_{i+1}^{i} \cup D_{i}^{i+1}\right)
$$

Pick $w \in \Gamma(r) \cap\left(D_{d}^{d} \cup D_{d}^{d+1}\right)$ and consider the set $C$ of $2 d$-cycles $\left(x_{0}=w, x_{1}=\right.$ $\left.r, x_{2}, \ldots, x_{2 d-1}, w\right)$ through $w r$. Note that at most $2 d-2$ edges of such a cycle have both endpoints in $A$. For $1 \leq i \leq 2 d-1$ let $C_{i}$ denote the subset of $C$ defined as follows. A cycle $\left(x_{0}=w, x_{1}=r, x_{2}, \ldots, x_{2 d-1}, w\right)$ is an element of $C_{i}$, if and only if $\left\{x_{1}, \ldots, x_{i}\right\} \subseteq A$ and $x_{i+1} \notin A$, where the addition in subscripts is computed modulo $2 d$. For example, cycles in $C_{1}$ are those $2 d$-cycles $\left(x_{0}=w, x_{1}=r, x_{2}, \ldots, x_{2 d-1}, w\right)$, for which $x_{2} \notin A$, while cycles in $C_{2 d-1}$ are those for which $\left\{x_{1}, x_{2}, \ldots, x_{2 d-1}\right\} \subseteq A$. Note that the sets $C_{i}$ are pairwise disjoint, and so

$$
a_{1} \leq n(w r) \leq\left|C_{1}\right|+\left|C_{2}\right|+\cdots+\left|C_{2 d-1}\right|
$$

Let us now estimate the above sum. To do this we introduce the following notation. For $i \in\{1,3, \ldots, 2 d-1\}$ we define

$$
\epsilon_{i}=\sum h(b)
$$

where the sum is over those vertices $b \in D_{d}^{d-1}$, for which $d(r, b)=i$. Note that

$$
\epsilon_{1}+\epsilon_{3}+\cdots+\epsilon_{2 d-1} \leq \epsilon
$$


We also define

$$
\kappa=\left|\Gamma(w) \cap\left(D_{d-1}^{d} \backslash\{r\}\right)\right|=\left|\Gamma(w) \cap D_{d-1}^{d}\right|-1 .
$$

Note that $\alpha+\kappa \leq \epsilon$.

Consider a $2 d$-cycle $\left(x_{0}=w, x_{1}=r, x_{2}, \ldots, x_{2 d-1}, w\right) \in C_{1}$. Observe that there are $\alpha-1$ choices for $x_{2}$. For each such choice of $x_{2}$, there are, by Lemma 2.6, at most $(k-1)^{d-1}$ girth-cycles containing both edges $w r$ and $r x_{2}$. Therefore,

$$
\left|C_{1}\right| \leq(\alpha-1)(k-1)^{d-1} .
$$

Consider a $2 d$-cycle $\left(x_{0}=w, x_{1}=r, x_{2}, \ldots, x_{2 d-1}, w\right) \in C_{2} \cup C_{4} \cup \cdots \cup C_{2 d-2}$. Assume that this cycle is an element of $C_{2 j}(1 \leq j \leq d-1)$. Observe that in this case we have that $x_{2 j} \in D_{d}^{d-1}$ and that $d\left(r, x_{2 j}\right)=2 j-1$ (otherwise there would be a cycle of length less than $2 d$ ). Therefore, we could choose an edge $x_{2 j} x_{2 j+1}$ in $\epsilon_{2 j-1}$ different ways. For each such choice of an edge $x_{2 j} x_{2 j+1}$, there are, by Lemma 2.6, at most $(k-1)^{d-2}$ girth-cycles containing edges $w r$ and $x_{2 j} x_{2 j+1}$, and so

$$
\left|C_{2}\right|+\left|C_{4}\right|+\cdots+\left|C_{2 d-2}\right| \leq\left(\epsilon_{1}+\epsilon_{3}+\cdots+\epsilon_{2 d-3}\right)(k-1)^{d-2} \leq \epsilon(k-1)^{d-2} .
$$

Consider a $2 d$-cycle $\left(x_{0}=w, x_{1}=r, x_{2}, \ldots, x_{2 d-1}, w\right) \in C_{3} \cup C_{5} \cup \cdots \cup C_{2 d-3}$. If this cycle is an element of $C_{2 j+1}(1 \leq j \leq d-2)$, then it is easy to see that $x_{2 j+1} \in D_{d-1}^{d}$, and so $x_{2 j+2} \in\left(D_{d}^{d} \cup D_{d}^{d+1}\right) \backslash\{w\}$. Therefore, there are at most $\epsilon-\kappa-\alpha$ choices for an edge $x_{2 j+1} x_{2 j+2}$. For each such choice there are, by Lemma 2.6 , at most $(k-1)^{d-3}$ girth-cycles containing edges $w r$ and $x_{2 j+1} x_{2 j+2}$, and so

$$
\left|C_{3}\right|+\left|C_{5}\right|+\cdots+\left|C_{2 d-3}\right| \leq(\epsilon-\kappa-\alpha)(k-1)^{d-3} .
$$

Finally, consider a $2 d$-cycle $\left(x_{0}=w, x_{1}=r, x_{2}, \ldots, x_{2 d-1}, w\right) \in C_{2 d-1}$. Note that we have at most $k-\alpha$ choices for a vertex $x_{2}$. For each choice of vertices $x_{2}, x_{3}, \ldots, x_{i-1}$, where $i \leq d$, we have at most $k-1$ choices for vertex $x_{i}$. Therefore, there are at most $(k-\alpha)(k-1)^{d-2}$ choices for vertices $x_{2}, x_{3}, \ldots, x_{d}$. On the other hand, there are at most $\kappa$ choices for a vertex $x_{2 d-1}$. For each such choice of vertices $x_{2}, x_{3}, \ldots, x_{d}$ and $x_{2 d-1}$, there is at most one girth-cycle containing the edges $w r, r x_{2}, x_{2} x_{3}, \ldots x_{d-1} x_{d}$ and $x_{2 d-1} w$. Therefore,

$$
\left|C_{2 d-1}\right| \leq \kappa(k-\alpha)(k-1)^{d-2} .
$$

To further estimate the sum $\left|C_{1}\right|+\left|C_{2}\right|+\cdots+\left|C_{2 d-1}\right|$, we first note that

$$
\begin{aligned}
\left|C_{1}\right|+\left|C_{2 d-1}\right| & \leq(k-1)^{d-3}\left((\alpha-1)(k-1)^{2}+\kappa(k-\alpha)(k-1)\right) \\
& =(k-1)^{d-3}\left((\alpha-1+\kappa)(k-1)^{2}-\kappa(\alpha-1)(k-1)\right) \\
& \leq(k-1)^{d-3}(\alpha-1+\kappa)(k-1)^{2} \\
& \leq(k-1)^{d-3}(\epsilon-1)(k-1)^{2},
\end{aligned}
$$

while

$$
\begin{aligned}
\left|C_{2}\right|+\left|C_{3}\right|+\cdots+\left|C_{2 d-2}\right| & \leq \epsilon(k-1)^{d-2}+(\epsilon-\kappa-\alpha)(k-1)^{d-3} \\
& =(k-1)^{d-3}(\epsilon(k-1)+\epsilon-\kappa-\alpha) \\
& \leq(k-1)^{d-3}(\epsilon(k-1)+\epsilon-1)=(k-1)^{d-3}(k \epsilon-1) .
\end{aligned}
$$


Therefore,

$$
\begin{aligned}
a_{1} \leq n(w r) & \leq\left|C_{1}\right|+\left|C_{2}\right|+\cdots+\left|C_{2 d-1}\right| \\
& \leq(k-1)^{d-3}\left((\epsilon-1)(k-1)^{2}+k \epsilon-1\right) \\
& =(k-1)^{d-3}\left(\epsilon\left(k^{2}-k\right)+\epsilon-(k-1)^{2}-1\right) \\
& <(k-1)^{d-3}\left(\epsilon\left(k^{2}-k\right)+(k-1)-(k-1)^{2}\right) \\
& \leq(k-1)^{d-2}(k \epsilon-k+2) .
\end{aligned}
$$

The result follows.

Theorem 4.3. With reference to Notation 2.7, assume that $g=2 d \geq 6$. Then $\epsilon=0$ and $\Gamma$ is either the incidence graph of a finite projective plane of order $k-1$, or the incidence graph of a finite generalized d-gon of order $(k-1, k-1)$. Hence in the latter case we have $d \in\{4,6,8\}$.

Proof. Suppose first that $\epsilon$ is positive. By Lemma 4.1 and 4.2 we have

$$
(k-1)^{d}-k \epsilon \leq a_{1}<(k-1)^{d-2}(k \epsilon-k+2) .
$$

This implies

$$
k-1>\epsilon>\frac{(k-1)^{d-2}\left(k^{2}-k-1\right)}{k\left(1+(k-1)^{d-2}\right)}=(k-2)+. \frac{(k-1)^{d-1}-k(k-2)}{k\left(1+(k-1)^{d-2}\right)} .
$$

As $k(k-2)<(k-1)^{2} \leq(k-1)^{d-1}$, the above inequality implies

$$
k-1>\epsilon>k-2,
$$

contradicting the fact that $\epsilon$ is an integer. Therefore, $\epsilon=0$. It is now easy to see that $\Gamma$ is isomorphic to the incidence graph of a finite projective plane of order $k-1$ if $d=3$, or the incidence graph of a generalized $d$-gon of order $(k-1, k-1)$ if $d \geq 4$ (a proof can be found in [11, Theorem 12.33]). By the famous theorem of Feit and Higman [6], we have that in the latter case $d \in\{4,6,8\}$.

\section{$5 \quad$ Examples}

In this section we provide examples shoving that the bound $\epsilon<k-1$ is tight in Theorem 3.6 for all $k$, it is sharp in Theorem 4.3 for some particular values of $k$ and close to the bound for some infinite families. These families arise from finite projective planes and generalized quadrangles. Let us start with the $g=4$ case.

Example 5.1. The complete bipartite graph minus a one-factor $K_{k+1, k+1}-(k+1) K_{2}$ is girth-regular with $g=4$ and $a_{1}=a_{2}=\cdots=a_{k}=(k-1)^{2}-(k-1)$.

In the case $g=6$ some graphs with small valency reach or are close to the bound $\epsilon=k-1$. These examples belong to the family of cyclic Haar graphs. For the detailed description of these graphs we refer to the book of Pisanski and Servatius [15]. It is known that the girth of these bipartite graphs is at most 6 (see [15, Theorem 5.26]). 
Example 5.2 (Cyclic Haar graphs). Let $0=d_{1}<d_{2}<\cdots<d_{k}=m$ and $1 \leq s$ be integers such that $d_{i+j}-d_{i}=d_{i^{\prime}+j^{\prime}}-d_{i^{\prime}}$ implies $i=i^{\prime}$ and $j=j^{\prime}$. Then the set $D=\left\{d_{1}, d_{2}, \ldots, d_{k}\right\}$ is a non-extendible almost difference set in $\mathbb{Z}_{2 m+s}$. Let $\Gamma$ be the Cayley graph on the dihedral group $D_{2 m+s}$ defined by the connection set $S=\left\{\operatorname{tr}^{d_{i}}: d_{i} \in D\right\}$. Then $\Gamma$ is a girth-regular graph on $2(2 m+s)$ vertices with $g=6$ and valency $k$.

Recall that an almost difference set with parameters $(v, k, \lambda, t)$ is a $k$-element subset $D$ in a group $G$ with $|G|=v$ such that the expressions $g h^{-1}, g, h \in D, g \neq h$ represent $t$ elements of $G$ exactly $\lambda$ times, and the remaining non-identity elements $\lambda+1$ times. So, in our case, $\lambda=0$. For almost difference sets, see the survey [12], and Chapter 5 of the book by Ding [4].

There is no 4-cycle in $\Gamma$, because $D$ is an almost difference set, hence

$$
\left(t r^{d_{i}} t r^{d_{j}}\right)\left(t r^{d_{i^{\prime}}} t r^{d_{j^{\prime}}}\right)=r^{\left(d_{j}-d_{i}\right)} r^{\left(d_{j^{\prime}}-d_{i^{\prime}}\right)}=r^{\left(d_{j}-d_{i}\right)-\left(d_{i^{\prime}}-d_{j^{\prime}}\right)} \neq \mathrm{id} .
$$

(i) For $k=3$ and 4 let $D=\{0,1,3\}$ and $\{0,1,4,6\}$, respectively. Then for $s=1$ we get the Levi graph of the projective plane of order 2 and 3, respectively.

For $s=2$ take the edge $1 t$ and construct $\Gamma$ and $\Gamma^{\prime}$, Then the two vertices in $\Gamma \backslash \Gamma^{\prime}$ are $r^{m+1}$ and $t r^{m+1}$, and they are adjacent because

$$
t r^{m+1} \cdot t=r^{-(m+1)}=r^{m+1} .
$$

Thus there are $1+2(k-1)$ edges in $\Gamma \backslash \Gamma^{\prime}$. So $\Gamma^{\prime}$ contains $(k-1)^{3}-(k-1)$ edges rb for which $d(1, r)=d(t, b)=2$, hence $a_{k}=(k-1)^{3}-(k-1)$.

(ii) For $k=3$ this is the generalized Petersen graph $G P(8,3)$, also called the MöbiusKantor graph. It is cubic girth-regular graph with girth 6 and signature $(6,6,6)$. Note that $6=8-2=(k-1)^{3}-(k-1)$.

(iii) For $k=4$ the graph $\Gamma$ is the Levi graph of a $\left(14_{4}\right)$ configuration. In this case $\Gamma$ is a tetravalent girth-regular graph on 28 vertices with signature $(24,24,24,24)$. Note that $24=27-3=(k-1)^{3}-(k-1)$.

(iv) For $k=5, D=\{0,1,3,7,12\}$ and $s=1$ we get a girth-regular graph on 50 vertices with signature $(50,50,50,51,51)$. Note that $a_{5}=51=(k-1)^{3}-(k-1)(k-2)-1$.

(v) For $k=6, D=\{0,1,3,7,15,20\}$ and $s=1$ we get a girth-regular graph on 82 vertices with signature $(81,87,88,88,92,92)$. Note that $a_{6}=92=(k-1)^{3}-(k-$ 1) $(k-2)-13$.

Unfortunately, cyclic Haar graphs have worse parameters for $k>6$.

Example 5.3. The unique $(7,6)$-cage $\Gamma$ has 90 vertices. This graph was first discovered by Baker [2], but it is usually named after O'Keefe and Wong [13]. It is a bipartite girth-regular graph with $a_{7}=204=(k-1)^{3}-2(k-1)$.

Take any edge $u v$ of $\Gamma$. Let $A=\{u, v\} \cup D_{2}^{1} \cup D_{1}^{2} \cup D_{3}^{2} \cup D_{2}^{3}$. Then $|A|=2(1+6+36)=86$. Let $x, y, w, z$ denote the four other vertices of $\Gamma$. We may assume without loss of generality that $x, y \in D_{3}^{4}$ and $w, z \in D_{4}^{3}$. The seven neighbours of $x$ must be in $\{w, z\} \cup D_{2}^{3}$. For any 
vertex $t \in D_{1}^{2}$ there is at most one path of length two joining $t$ and $x$, otherwise a cycle of length four would appear. Hence at least one neighbour of $x$ must be in $\{w, z\}$. It is easy to see (or check by computer), that exactly one neighbour of $x$ is in $\{w, z\}$. The same argument shows that exactly one neighbour of $y$ is in $\{w, z\}$, and exactly one neighbour of $w$ and $z$ is in $\{x, y\}$.

Thus, by Proposition 2.5 (vi), we have $\epsilon=2 \cdot 6=2(k-1)$. As $u v$ was an arbitrary edge, we also proved that the signature of $\Gamma$ is $(204,204, \ldots, 204)$.

Infinite families of graphs having $\epsilon \approx(k-1)^{2}$ can be constructed by removing subsets of points and lines from projective planes. These methods work when $k=q$, a prime power. The next two constructions were originally due to Abreu et al. [1], their geometric description was given by Gács and Héger [8].

Example 5.4. If we remove a pencil $\mathcal{P}$ of $k+1$ lines and the carrier $P$ of $\mathcal{P}$ from an affine plane of order $k$, then we get a truncated projective plane. Its incidence graph is a girth-regular graph on $2\left(k^{2}-1\right)$ vertices with $g=6$, valency $k$, and signature $a_{1}=a_{2}=$ $\cdots=a_{k-1}=(k-1)^{3}-(k-1)(k-2)$.

For any incident point-line pair $(R, \ell)$ there are $(k-1)^{2}$ points which are collinear with $R$ but are not incident with $\ell$. Let $T$ be one of these points. Then there are $k$ lines through $T$, one of them is $R T$. There are two possibilities for the remaining $k-1$ lines:

Case 1. If $P T$ is not parallel to $\ell$, then one of these lines is parallel to $\ell$, each other line intersects $\ell$ in a unique point. There are $(k-1)(k-2)$ choices for $T$, thus we get $(k-1)(k-2)^{2}$ girth-cycles.

Case 2. If $P T$ is parallel to $\ell$, then each of these lines intersects $\ell$ in a unique point. There are $k-1$ choices for $T$, thus we get $(k-1)^{2}$ girth-cycles in this case.

So the total number of 6 -cycles containing the edge corresponding to the pair $(P, \ell)$ is

$$
(k-1)(k-2)^{2}+(k-1)^{2}=(k-1)^{3}-(k-1)(k-2) .
$$

Let us remark that for $k=3$ this construction results in the same graph as Example $5.2(i i)$.

Example 5.5. Suppose that $k$ is a square and a projective plane $\Pi$ of order $k$ has a Baer subplane $\mathcal{B}$ of order $\sqrt{k}$. Remove the points and lines of $\mathcal{B}$ from $\Pi$. (For example, if $k=q=p^{2 h}$, p prime, we can take $\Pi$ as $\mathrm{PG}(2, q)$.) The incidence graph of the remaining structure is a girth-regular graph on $2\left(k^{2}-\sqrt{k}\right)$ vertices with $g=6$, valency $k$, and signature $a_{1}=a_{2}=\cdots=a_{k-1}=(k-1)^{3}-(k-1)(k-\sqrt{k}-1)$.

Take any incident point-line pair $(P, \ell)$ in $\Pi \backslash \mathcal{B}$. In $\Pi$ there is a unique point $T=\ell \cap \mathcal{B}$. In $\Pi \backslash \mathcal{B}$ there are $(k-1)^{2}$ points which are collinear with $P$ but are not incident with $\ell$. Let $R$ be one of these points. Then in $\Pi$ there are $k$ lines through $R$ other than $P R$. One of them is $R T$ and one of them is a $\sqrt{k}$-secant of $\mathcal{B}$. These two lines are the same for $(\sqrt{k}+1)(k-\sqrt{k})$ choices of $R$ when $R T$ is a secant of $\mathcal{B}$ in $\Pi$, otherwise we get two distict lines.

So the total number of 6 -cycles containing the edge corresponding to the pair $(P, \ell)$ in $\Pi \backslash \mathcal{B}$ is

$$
(k-1)^{2}(k-2)+(\sqrt{k}+1)(k-\sqrt{k})=(k-1)^{3}-(k-1)(k-\sqrt{k}-1) .
$$


Let us remark that for $k=4$ this construction yields the same graph as Example $5.2($ iii $)$.

Example 5.6. We get a biaffine plane of order $k$ if we remove a parallel class of lines from an affine plane of order $k$. The incidence graph of a biaffine plane of order $k>2$ is a girth-regular graph on $2 k^{2}$ vertices with $g=6$, valency $k$, and signature $a_{1}=a_{2}=\cdots=$ $a_{k}=(k-1)^{3}-(k-1)^{2}$.

For any incident point-line pair $(P, \ell)$ there are $(k-1)^{2}$ points which are collinear with $P$ but are not incident with $\ell$. Let $R$ be one of these points. Then there are $k$ lines through $R$, one of them is $P R$ and one of them is parallel to $\ell$, each other line intersects $\ell$ in a unique point. Thus in $\Gamma$ the number of 6 -cycles containing the edge corresponding to the pair $(P, \ell)$ is

$$
(k-1)^{2}(k-2)=(k-1)^{3}-(k-1)^{2} .
$$

In the case $g=8$ our example comes from the incidence graph of a generalized quadrangle. For a detailed descriptions of generalized quadrangles, their ovoids and spreads we refer the reader to the book of Payne and Thas [14].

Example 5.7. Let $\mathcal{G}=(\mathcal{P}, \mathcal{L}, \mathrm{I})$ be a generalized quadrangle of order $k$ which admits both an ovoid $\mathcal{O}$ (a set of $k^{2}+1$ points, no two of them are collinear) and a spread $\mathcal{S}$ (a set of $k^{2}+1$ lines, no two of them intersecting). Delete the points of an ovoid and the lines of a spread. Then the Levi graph of $(\mathcal{P} \backslash \mathcal{O}, \mathcal{L} \backslash \mathcal{S}, \mathrm{I})$ is a girth-regular graph on $2 k\left(k^{2}+1\right)$ vertices with $g=8$, valency $k$ and $a_{k}=(k-1)^{4}-(2 k-3)(k-1)^{2}$.

For any incident point-line pair $(P, \ell)$ there are $(k-1)^{2}$ points in $\mathcal{P} \backslash \mathcal{O}$ which are collinear with $P$ but are not incident with $\ell$, and there are $(k-1)^{2}$ lines in $\mathcal{L} \backslash \mathcal{S}$ which meet $\ell$ but are not incident with $P$.

Let $R$ be one of these points and $e$ be one of these lines. Then there is a unique point-line pair $(T, f)$ in $\mathcal{G}$ such that $R \mathrm{I} f \mathrm{I} T \mathrm{I} e$. It could happen, that $T \in \mathcal{O}$ or $f \in \mathcal{S}$ or both. Among these $(k-1)^{4}$ girth-cycles there are $(k-1)^{2}(k-1)$ for which $f \in \mathcal{S}$ (there is a unique element of $\mathcal{S}$ through $R$, and no two elements of $\mathcal{S}$ intersect each other), and dually, there are $(k-1)^{2}(k-1)$ girth-cycles for which $T \in \mathcal{O}$. Each element of $\mathcal{S}$ incident with a unique element of $\mathcal{O}$, so there are $(k-1)^{2}$ cycles with $f \in \mathcal{S}$ and $T \in \mathcal{O}$. Thus the total number of girth-cycles through $(P, \ell)$ in $(\mathcal{P} \backslash \mathcal{O}, \mathcal{L} \backslash \mathcal{S}, I)$ is

$$
(k-1)^{4}-2(k-1)^{2}(k-1)+(k-1)^{2}=(k-1)^{4}-(2 k-3)(k-1)^{2} .
$$

Among the known generalized quadrangles only one admits both an ovoid and a spread, this is $W(q)$ for $q$ even. So this example gives graphs when $k=2^{h}$ and $h>1$.

It might be tempting to conjecture a bound of type $(k-1)^{d}-c(k-1)^{d-1}, c \approx 1$, but Examples 5.2 and 5.3 show that such a result can only be expected for $k$ large enough.

Finally, let us remark that we cannot expect results similar to our main theorem if the girth is odd. In these cases, by Proposition 2.3, the maximum number of girth-cycles compared to the number of vertices is much less than in the even girth case.

- For $g=3$ and $k=2 m$ we have $a_{k} \leq 2 m-2$ and the complete graph minus a one-factor $K_{2 m}-m K_{2}$ is girth-regular with $a_{1}=a_{2}=\cdots=a_{k}=2 m-4$, hence $\epsilon=2$. 
- For $g=5$ and $k=6$ the $(6,5)$-cage (see [20]) is girth-regular with $a_{1}=a_{2}=\cdots=$ $a_{k}=22=(k-1)^{2}-3$, hence $\epsilon=3$.

\section{References}

[1] M. Abreu, M. Funk, D. Labbate, V. Napolitano, On (minimal) regular graphs of girth 6, Australas. J. Combin, 35 (2006), 119-132.

[2] R. D. Baker, An elliptic semiplane, J. Combin Theory Ser. A 25 (1978), 193-195.

[3] B. Bollobás, Extremal Graph Theory, Dover Books on Mathematics, 1978.

[4] C. Ding, Codes from Difference Sets, World Scientific, Singapore, 2014

[5] G. Exoo, R. Jajcay, Dynamic Cage Survey, Electron. J. Combin., dynamic survey DS16

https://www.combinatorics.org/ojs/index.php/eljc/article/view/DS16/pdf

[6] W. Feit and G. Higman, The non-existence of certain generalized polygons, J. Algebra 1 (1964), 114-131.

[7] Z. Füredi, M. Simonovits, The History of Degenerate (Bipartite) Extremal Graph Problems, in: (L. Lovász, I. Z. Ruzsa, V. T. Sós eds.) Erdôs Centennial, Bolyai Society Math. Studies 25, 169-264, Springer, 2013.

[8] A. Gács, T. Héger, On geometric constructions of $(k, g)$-graphs, Contrib. Discrete Math. 3 (2008), 63-80.

[9] R. Jajcay, Gy. Kiss and Š. Miklavič, Edge-girth-regular graphs, European J. Combin. 72 (2018), 70-82.

[10] F. Kárteszi, Piani finiti ciclici come risoluzioni di un certo problema di minimo, Boll. Un. Mat. Ital. 15 (3) (1960), 522-528.

[11] Gy. Kiss, T. Szônyi, Finite Geometries, CRC Press, Taylor \& Francis Group, 2019.

[12] K. Nowak, A Survey on Almost Difference Sets, arXiv:1409.0114

[13] M. O'Keefe, P. K. Wong, The smallest graph of girth 6 and valency 7, J. Graph Theory, 5 (1981), 79-85.

[14] S. E. Payne, J. A. Thas, Finite Generalized Quadrangles, Second Ed., EMS Publishing House, 2009.

[15] T. Pisanski, B. Servatius, Configurations from a Graphical Wievpoint, Birkhäuser, 2013.

[16] M. Miller, J. Sirán, Moore Graphs and Beyond: A survey of the Degree/Diameter Problem, Electron. J. Combin., dynamic survey DS14 https://www.combinatorics.org/ojs/index.php/eljc/article/view/DS14/pdf 
[17] P. Potočnik and J. Vidali, Girth-regular graphs, Ars Math. Contemp. 17 (2019), 349-368.

[18] M. Simonovits, Paul Erdôs's Influence on Extremal Graph Theory, in: The Mathematics of Paul Erdôs II Algorithms and Combinatorics Volume 14, 1997, 148-192, updated version on Miklós Simonovits's homepage:

https://www.renyi.hu/ miki/SimonovitsX.pdf

[19] M. Simonovits, Paul Turán's Influence in combinatorics, in: Number theory, analysis, and combinatorics, 309-392, De Gruyter Proc. Math., De Gruyter, Berlin, 2014.

[20] P. K. Wong, On the uniqueness of the smallest graphs of girth 5 and valency $6, J$. Graph Theory, 3 (1982), 1-22.

György Kiss: Department of Geometry and MTA-ELTE Geometric and Algebraic Combinatorics Research Group, Eötvös Loránd University, 1117 Budapest, Pázmány s. 1/c, Hungary; and Faculty of Mathematics, Natural Sciences and Information Technologies, University of Primorska, Glagoljaška 8, 6000 Koper, Slovenia; e-mail: kissgy@cs .elte.hu

Štefko Miklavič: Andrej Marušič Institute, University of Primorska, Muzejski trg 2, 6000 Koper; and Institute of Mathematics, Physics and Mechanics, Jadranska 19, 1000 Ljubljana, Slovenia; e-mail: stefko.miklavic@upr.si

Tamás Szőnyi: Department of Computer Science and MTA-ELTE Geometric and Algebraic Combinatorics Research Group, Eötvös Loránd University, 1117 Budapest, Pázmány s. 1/c, Hungary; and Faculty of Mathematics, Natural Sciences and Information Technologies, University of Primorska, Glagoljaška 8, 6000 Koper, Slovenia;

e-mail: szonyi@cs.elte.hu 